\title{
Towards early detection of retinoblastoma
}

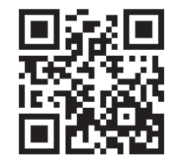

Retinoblastoma (RB) is a rare but life-threatening condition. If it is managed optimally by a competent health team there is an excellent prognosis for life, with survival rates of $95 \%$ in developed countries. Moreover, good visual outcomes are possible.

Kruger et al. ${ }^{[1]}$ in this issue of $S A M J$ have shown survival rates in a region of South Africa (SA) of only $50 \%$, reflecting the high frequency of late presentation, the simple reason for which is lack of effective screening. Early detection of suspected RB would significantly reduce this unacceptably high mortality rate.

Early detection may be achieved by performing a simple clinical test on all newborns and toddlers: the red reflex. The red reflex or retinal reflex refers to the reddish-orange reflection of light from normal retina, and it is observed with a direct ophthalmoscope held close to the examiner's eye, while observing the patient's eyes from a distance of approximately $30 \mathrm{~cm}$. Normally, the reflections of the two eyes are equivalent in colour, intensity and clarity. The examiner is looking for the presence of a whitening of the reflex, white spots in the reflex, an absent red reflex or asymmetry of the two red reflexes when viewed from various angles. ${ }^{[2,3]}$

It is considered standard of care that all newborn babies are tested for a red reflex at discharge from the neonatal nursery. Parents are similarly able to detect a problem by noticing an abnormality in the red reflex in photographs taken when the 'red eye reduction' function happens to be switched off on their camera. It would not be too idealistic to suggest that all doctors attending to neonates and toddlers use this humble camera technique and digitally save the red reflex images to the patient's file at each visit.

In the not-too-distant future, neonatal screening will include universal digital retinal imaging. The roll-out of diabetic screening programmes using portable digital imaging systems is already established in many parts of the world, including the Cape Town metropole. These systems could be expanded to also serve as screening units for neonates.

'Should eye imaging be part of a standard newborn examination?' was the theme of a session presented at the 2014 World Ophthalmology Congress of the International Council of Ophthalmology on 3 April 2014 in Tokyo, Japan. Speakers from the USA, Russia, India, China and Taiwan shared their experience with modern retinal imaging systems used in universal eye imaging programmes in their countries, convincing the audience about the value of such systems in neonates. The RetCam (Clarity Medical Systems, USA) is an example of a medical device utilising innovative 21st-century optical, electronic and information technologies to image the retina of neonates. Its ability to perform wide-field imaging on neonatal retinas has revolutionised neonatal retinal disease management in the developed world. It has been shown to be a safe screening tool when operated by trained technicians. ${ }^{[4]}$ Owing to the current cost of this technology, it is not yet realistic to propose universal digital retinal imaging of all neonatal retinas in SA. However, the development of portable digital retinal imaging systems is ongoing, and it will be feasible to screen the retinas of all neonates in the future using this technology.

While we were mulling over this editorial, a 3-month-old boy presented with bilateral RB. The father had had one eye enucleated by us for RB 25 years ago. Patients who were treated decades ago may have forgotten that their children are at risk, and they may not be aware that genetic testing and/or counselling can now be offered. Once a heritable mutation is identified, molecular testing can determine which members of a family are at risk of developing RB. Children who carry the mutation need to be screened clinically by an ophthalmologist 1 week after birth, monthly for 3 months, every 2 months for 6 months, every 3 months for 2 years and then every 6 months. This is initially best achieved under general anaesthesia until the child is co-operative enough for retinal examination while awake. This approach allows for early detection of small tumours. Children who do not carry the family's RB1 mutation are not at increased risk and do not require repeated retinal examinations. The benefits and cost-effectiveness of genetic screening in affected RB families have been well demonstrated. ${ }^{[5]}$

All the modern ophthalmological treatment modalities for RB are available in several centres in SA. For its size and population, SA has an adequate number of these tertiary centres and even boasts a quaternary centre offering radiation plaque therapy ${ }^{[6]}$

Appropriate and timely treatment can be simultaneously curative and vision preserving. Since management of a child diagnosed with $\mathrm{RB}$ requires a multidisciplinary approach, affected children are best referred to tertiary/quaternary facilities that provide specialist paediatric ophthalmology and oncology services. The various provincial departments of health need to co-operate and ensure referral of these patients to the existing treatment centres.

As a country we can be proud that our health system has the expertise to manage a child with RB well. The issue at stake is timely referral of the affected child to one of the specialist treatment centres. Our national mortality and morbidity figures for this malignancy will only improve if universal eye screening proves successful at primary care level. ${ }^{[7,8]}$ Until universal screening with digital imaging becomes a reality, the tried-and-trusted, old-fashioned red reflex test should be mandatory at discharge from all neonatal services and at all subsequent routine health supervision visits. Most RBs would then be detected early. An added benefit of compliance with such a screening regimen would be the detection of other serious vision-threatening disorders such as cataracts, glaucoma and eye muscle imbalances.

\section{Nicola Freeman \\ David Meyer \\ Division of Ophthalmology, Faculty of Medicine and Health Sciences, Stellenbosch University, Tygerberg, Cape Town, South Africa}

Corresponding author: D Meyer(dm2@sun.ac.za)

1. Kruger M, Reynders D, Omar F, Schoeman J, Wedi O, Harvey J. Retinoblastoma outcome at a single 2. Minnesota Department of Health, Vision Screening Online Training Program, Module 5: Retin Reflex (Red Reflex). http://www.health.state.mn.us/divs/fh/mch/webcourse/vision/mod5a.cfm (accessed 27 October 2014).

3. American Academy of Pediatrics, Section on Ophthalmology, American Association for Pediatric Ophthalmology and Strabismus, American Academy of Ophthalmology, American Associatio of Certified Orthoptists. Red reflex examination in neonates, infants, and children. Pediatrics of Certified Orthoptists. Red reflex examination in neonates, in
2008;122(6):1401-1404. [http://dx.doi.org/10.1542/peds.2008-2624]

4. Vinekar A, Gilbert C, Dogra M, et al. The KIDROP model of combining strategies for providing retinopathy of prematurity screening in underserved areas in India using wide-field imaging, teleretinopathy of prematurity screening in underserved areas in India using wide-field imaging, tele-
medicine, non-physician graders and smart phone reporting. Indian J Ophthalmol 2014;62(1):41-49. medicine, non-physician graders and smart p
[http://dx.doi.org/10.4103/0301-4738.126178]

5. Joseph B, Shanmugam MP, Srinivasan MK, Kumaramanickavel G. Retinoblastoma: Genetic testing versus conventional clinical screening in India. Mol Diagn 2004;8(4):237-243. [http://dx.doi. org $/ 10.2165 / 00066982-200408040-00005$

6. Stannard C, Sauerwein W, Maree G, Lecuona K. Radiotherapy for ocular tumours. Eye 2013;27(2):119127. [http://dx.doi.org/10.1038/eye.2012.241]

. Leander C, Fu LC, Peña A, et al. Impact of an education program on late diagnosis of retinoblastoma in

Honduras. Pediatr Blood Cancer 2007;49(6):817-819. [http://dx.doi.org/10.1002/pbc.21052]

8. Epelman S. Preserving vision in retinoblastoma through early detection and intervention. Curr Oncol

Afr Med J 2014;104(12):856. DOI:10.7196/SAMJ.8741 